\title{
Automotive power window system design: object-oriented modelling and design of experiments integration within a digital pattern approach
}

\author{
Stanislao Patalano ${ }^{a}$, Ferdinando Vitolo and Antonio Lanzotti \\ University of Naples Federico II, Department of Industrial Engineering, P.le V. Tecchio 80, 80125 Naples, Italy
}

Received 31 May 2015, Accepted 6 November 2015

\begin{abstract}
The paper tackles an integrated use of object-oriented modelling (OOM) and design of experiments (DoE) to determine the performance of a double bowden power window system in order to accomplish the expected system performances during the preliminary designing phase and, therefore, by accelerating the product development process. A DoE has been carried out by using the power window model, developed in Dymola environment. The response surface method (RSM) and ANOVA analysis are used to identify the significant factors. Then, an empirical equation is derived. It predicts the main system response i.e. the stroke time, expressed as dependency related to the significant factors. Finally, the use of the empirical relation inside a design flow of a power window, based on digital patterns, is presented.
\end{abstract}

Key words: Mechatronic systems / digital pattern for product development / object-oriented modeling / design of experiments / power window systems

\section{Introduction}

A mechatronic system could be defined as an "engineering system with mechanical, electrical, hydraulic or pneumatic components, including interactions of physical effects from various energy domains" [1]. Therefore, such system usually needs for a team of designers with different competencies and expertise due to its multi-disciplinary nature. As a consequence, adequate design models are expected to manage such concurrent contributions. Furthermore, the growing complexity of a mechatronic system, occurred since 1980s, implied the need to manage large and heterogeneous product data set, characterising product models. For these reasons [2], first and, then [3], state the existence of two classes of issues within mechatronic designing: the "Process-based problems" and the "Design data-related problems" i.e. the issues related to design methods and product model, respectively.

As regards the first, several authors worked on integrated methods. Chhabra and Reza Emami [3] face the possibility to design a mechatronic system as a whole. In particular, they adopt bond graph representations to consider mechatronic systems as energy systems and to define three holistic design criteria (namely energy, entropy

${ }^{a}$ Corresponding author: patalano@unina.it and agility) within the designing of the mechatronic system. In particular, each criterion has to be used when the power energy, the thermodynamic aspects or the operative aspects of the system, respectively, are the most significant. In reference [4] the author faces the relation between design methods and product model illustrating how hierarchical modeling could describe product models and data related to different disciplines involved in mechatronic system design, in order to correctly manage the interaction between design phases and related models. As regards the second, i.e. the issue related to product model, a secure need for a mechatronic system is represented by the possibility to create, manage and storage product data during the whole product lifecycle. Actually, this is accomplished by a series of proposed product data models such as STEP (STandard for the Exchange of Product model data) or CPM (Core Product Module). Such data models could support mechatronic system design processes but not in a complete way $[3,5]$.

Even if the design methods and product model represent two pillars within the designing of a mechatronic system a secure "bridge" between them i.e. an overlapping field is represented by multidisciplinary modelling and simulation. In fact, as a mechatronic system is characterised by an increasing "synergistic integration of physical systems with information technology and complex 
decision-making in the design, manufacture and operation of industrial products and processes" [6], the modelling and simulation of mechatronic systems integrating mechanical, electrical, electronic, thermal, control, fluid power, electric power or process-oriented components are significant tasks. Therefore, the object-oriented multidisciplinary modelling (OOM) and related languages are today widely used [7]. In fact, the possibility to explore different design solutions is accomplished by switching between domains (e.g. mechanical and controller domains) and comparing several design changes [8].

OOM and multi-domain tools based on Modelica language are very used by industrial companies that usually do not provide a deep insight into modelling and simulation but take advantages from the availability of wide range of libraries. As a consequence, dangerous conditions are represented by the lack of a validation phase and a high degree of complexity for the developed models [9]. For these reasons, methodological tools are necessary to reduce times and improve targets of designing activities.

Some large companies, involved in designing and manufacturing of automotive and railway means of transportation, introduced "digital patterns for product development" viz. geometrical data and models, to be reconfigured and re-used in different but similar design activities $[10,11]$. Such patterns are supported by a series of software tools, belonging to a decision support system, able to guarantee well-defined levels of product quality as well as the fulfilment of validating procedure to limit the overall manufacturing costs. Examples of such tools, operating with digital patterns, are "quality and standard checkers", "predictive engineering wizards" and "cost advisors". Therefore, OOM could be used to build up tools belonging to the decision support system and guide the project team during design activities. Further tools within the decision support system could be Design of Experiments (DoE), RSM and Robust Design. In fact, identification of the significant factors addressing the main system responses, the identification of meta model useful for numerical experiments and the definition of noise factors, that can affect the performance of the system during the whole life cycle, could allow understanding the origin of variation, optimizing performances and improving the robustness at operational level.

A power window system is a mechatronic system usually developed in automotive field. General methodologies were proposed, along the "process based problem", for example, dealing with the design of the power window controller [12] or the development of pinch detection algorithm, related to control system [13].

The paper is arranged as follows. Section 2 presents the approach and simulation tools used to accomplish the simulations of the power window system. Section 3 presents the architectures of automotive power window systems. Section 4 summarizes the developed object models while Section 5 illustrates the response surface method (RSM) adopted. Finally, Sections 5 and 6 draw results and conclusions, respectively.

\section{OOM and DoE as tools of the support decision system in a digital pattern approach to design}

Being the design of means of transportation an evolutionary rather than revolutionary task, engineering teams are involved in managing and modifying models and data previously used. Designers, in fact, are continuously engaged in the development of new products but they must re-use, as far as possible, previous models, data, and procedures. In fact, the improving of product quality as well as the reducing of manufacturing time and costs are strategic goals in a highly competitive and worldwide context. For these reasons, it is essential to re-use company knowledge embedded in models and numerical data by defining product and process digital patterns (DPs). Generally, such DPs, related to product and process, are geometrical and numerical models, preconfigured and parametrical, able to be easily adapted according to a set of constraints coming from a new designing task. The searching, use and storage of such DPs also represent a general methodology to be continuously applied within the company, to produce growing and positive effects in the time. International standards and company rules (Fig. 1) are initial content of DPs. Then, the development process is carried on thanks to parametrical CAD models that are cyclically linked to a decision support system. Finally, designers' choices are supported by a graphical user interface (GUI). Therefore, the digital pattern approach allows designers to collect, cumulate and share company knowledge as well as to easily accomplish verifications.

According to a digital pattern approach to designing OOM and DoE could be used as tools of the decision support system. From one side, OOM and related simulations, carried out for example in a Dymola-Modelica environment, provide results in terms of dimensioning of components and relative positioning; in such a way, designers are able to provide quick verification of different designing solutions because they verify the effects of several choices on the assembly in which the mechatronic system is assembled. On the other side, DoE is used to address the more significant factors related to a specific response of the system. RSM is also used to define an empirical model that can be used as acceptable approximation of the system response related to a given set of levels for each factor.

\section{Architectures of automotive power window systems}

Power window systems are electromechanical devices used to lift and lower the car window. Today, the systems are assembled in all front automotive doors. Several side rear doors are also equipped with such systems.

The architectures of a power window system are essentially four: cable spiral, pantograph, single bowden and, finally, double bowden (Fig. 2). Cable spiral and pantograph types are today not widely used. Single and 
S. Patalano et al.: Mechanics \& Industry 17, 505 (2016)

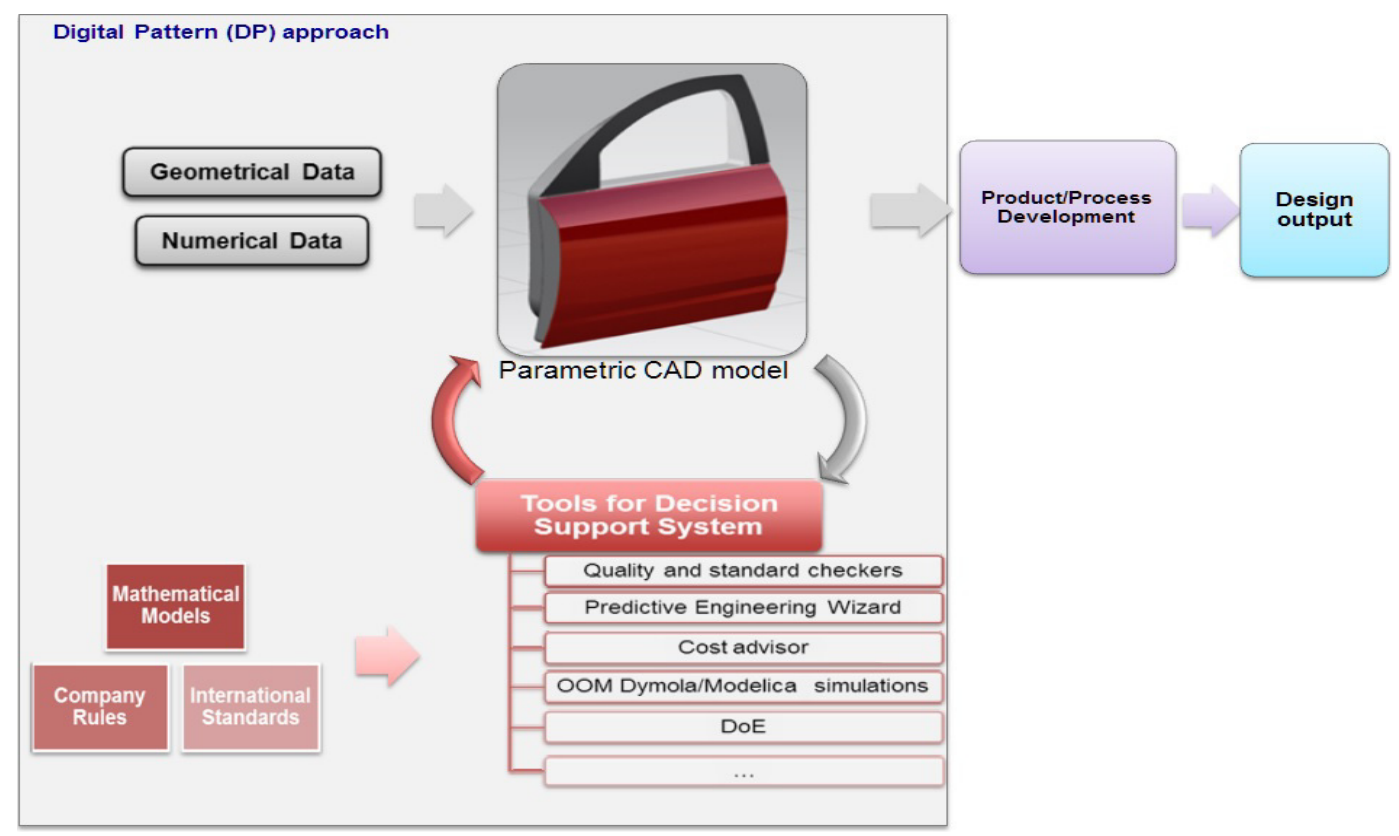

Fig. 1. Operational flow and related contents of a DP approach for product/process development.
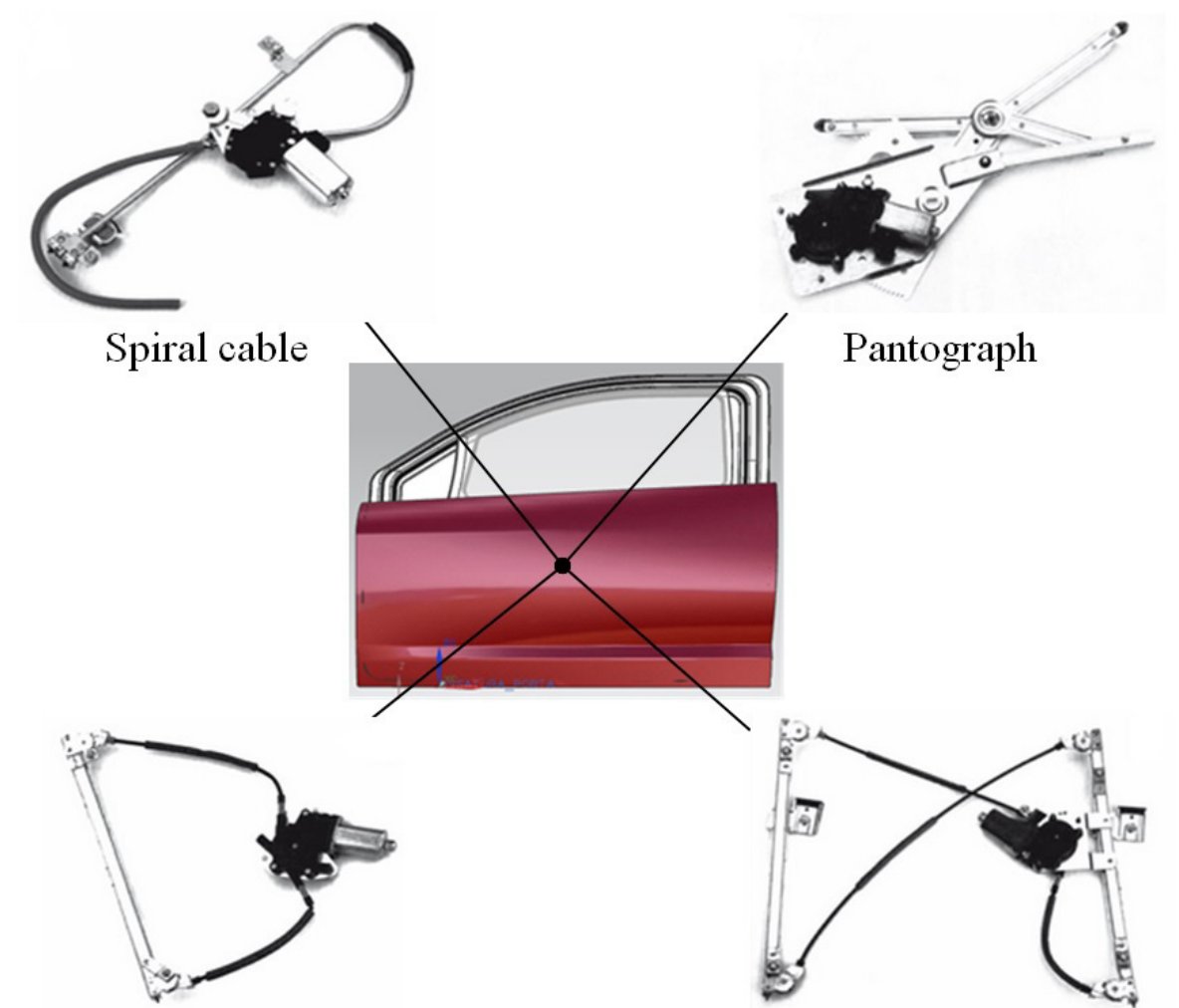

Pantograph

Single bowden

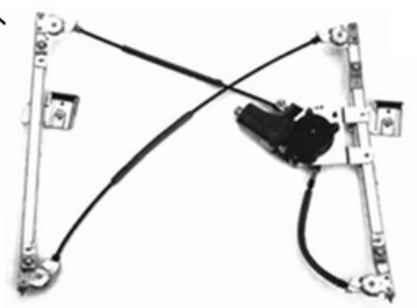

Double bowden

Fig. 2. Main architectures for power window systems. 


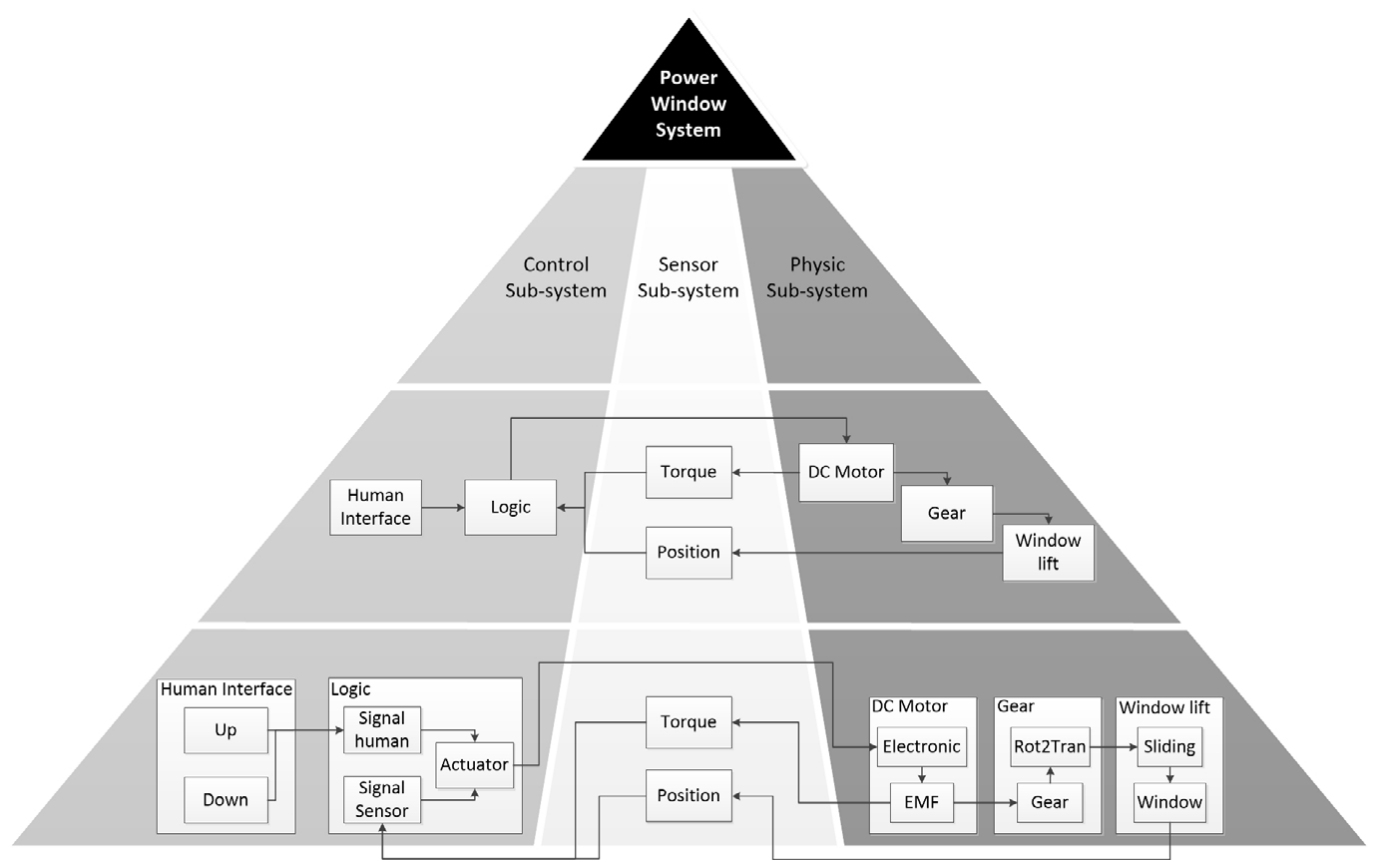

Fig. 3. Functional levels related to power window system modeling using a top-down approach.

double bowden types are generally used for short/light and long/heavy windows, respectively. The main subsystems/parts of such power windows systems are a DC motor, equipped with a worm gear, a sliding mechanism and a window constrained along two rails. The DC motorworm gear assembly is usually named "gear-motor" while the sliding mechanism usually characterizes the architecture of the power window. When the command button is moved upwards or downwards, a voltage with direct or reverse polarity is applied to motor, respectively. Therefore, the voltage drives the DC motor towards one or the other rotation. The motor moves a worm gear that wraps bowden cables by means a pulley. Then, the cable moves one or two supports and, consequently, lifts and lowers the automotive window, as it is equipped with one or two supports. We have single or double bowden type when cable shapes a single loop or double loop (cross cable), respectively (Fig. 2).

The present work deals with double bowden power window systems.

\section{Object models of the power window system}

The power window system is modelled by using a top down approach to OOM. Therefore, the modelling is accomplished by firstly providing a series of levels of detail (Fig. 3). The power window system is divided into three subsystems (first level decomposition) related to Control, Sensor and Physic subsystem, respectively. Such decomposition is also generally used tackling the design of mechatronic systems [14]. After this, each subsystem is decomposed according to functional groups i.e. by using a second level decomposition. Finally, the third level decomposition is related to generalised components i.e. components that characterize different physics such as physical parts or torque and force vectors or signals.

A primary need is to evaluate how physical components affect system behaviour. Therefore, the model related to the Physic subsystem is taken into account. As the power window behaviour is affected by control system [15], Control and Sensor subsystems are subsequently modelled and simulated by using the set of parameter defined for the physical sub-system.

The power window Physic system is modelled using Modelica language within Dymola environment. Six objects compose the model:

- $\mathrm{dC}$ motor object - it is characterized by three parameters: resistor, inductor and electromagnetic constant;

- gear object - it is characterized by two parameters: speed ratio and efficiency. As for DC motor in power window is generally used a worm gear efficiency value equal to $45 \%$;

- inertia object - it represents the inertia of all rotational components;

- pulley object - it is characterized by pulley radius;

- window object - it is modelled as a rectangular trapezoid without double curvature of window. Only the magnitude in the window movement direction is taken into account; window mass center is computed by means of Varignon's Theorem;

- mass object - it represents the window mass.

The power supply is modelled as a constant voltage generator $13 \mathrm{~V}$. At the beginning, no control on the evolution of the system is taken into account. The window stroke equal to $0.40 \mathrm{~m}$ is limited by means of a source code. Therefore, the simulation stops when the stroke is completed. This 


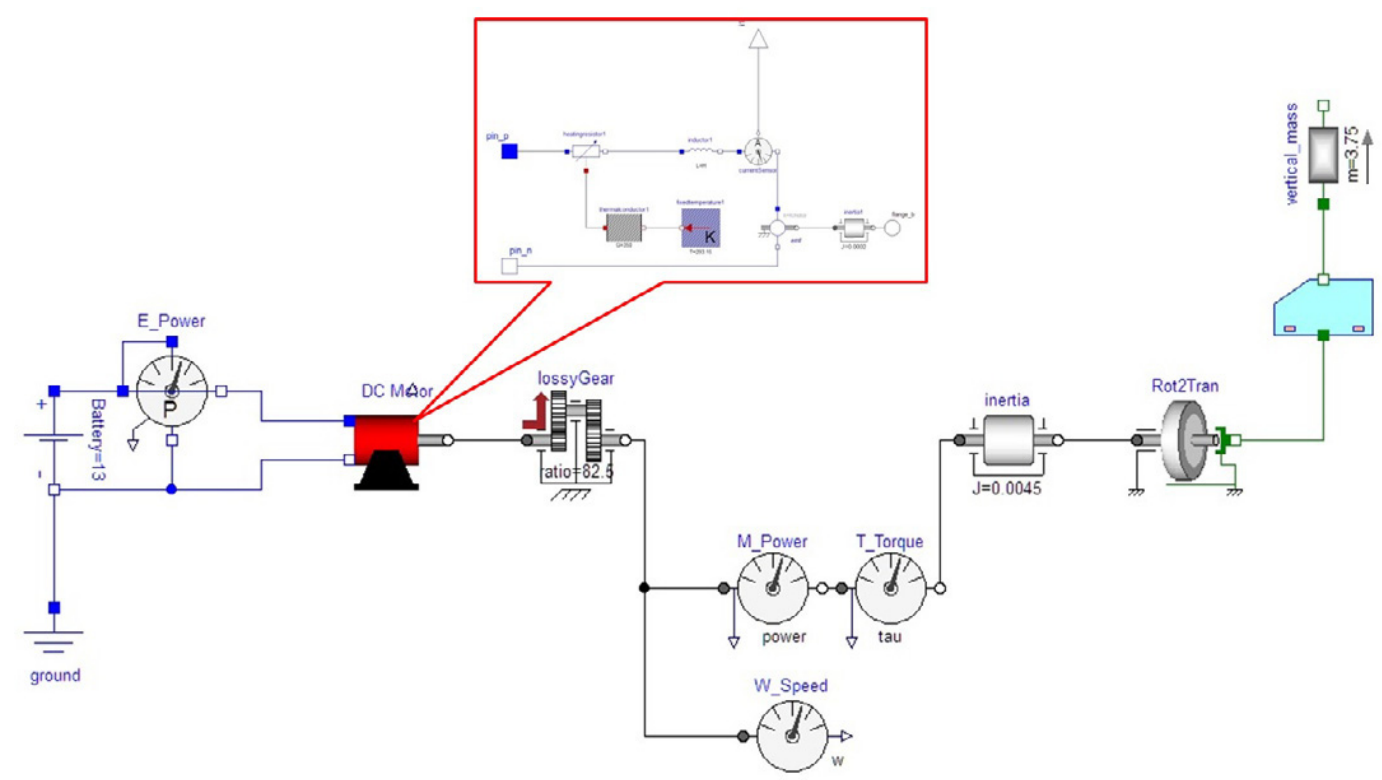

Fig. 4. Physic system model of power window within Dymola environment.

Table 1. Independent and dependent parameters in the model of power window system.

\begin{tabular}{|c|c|c|c|c|c|c|}
\hline & \multicolumn{4}{|c|}{ Independent parameters } & \multicolumn{2}{|c|}{ Dependent parameters } \\
\hline \multirow{5}{*}{$\begin{array}{c}\text { Gear } \\
\text { DC motor } \\
\text { Sliding } \\
\text { mechanism } \\
\text { Window }\end{array}$} & Speed ratio & Pulley diameter & Inertia & Efficiency & Torque & utput \\
\hline & Resistor & Inductor & Electromagnetic constant & Inertia & Current & Torque \\
\hline & & Inertia & Friction & & Force on & Force on \\
\hline & & & & & front support & rear support \\
\hline & & Weight & Stroke & & Displacement & Stroke time \\
\hline
\end{tabular}

Table 2. Levels of the factors $(\alpha=1.62658)$.

\begin{tabular}{lcccccc}
\hline & & $\alpha$ & -1 & 0 & +1 & $+\alpha$ \\
\hline $\mathrm{A}$ & Window weight $[\mathrm{kg}]$ & 2.00 & 2.77042 & 4.00 & 5.22958 & 6.00 \\
$\mathrm{~B}$ & Speed ratio & 55 & 65.5933 & 82.50 & 99.4067 & 110 \\
$\mathrm{C}$ & Pulley radius [m] & 0.02 & 0.0257782 & 0.035 & 0.0442218 & 0.05 \\
$\mathrm{D}$ & Inertia [Kg.m $\left.^{2}\right]$ & 0.001 & 0.00215564 & 0.004 & 0.00584436 & 0.007 \\
$\mathrm{E}$ & Electromagnetic constant [N.m/A] & 0.01 & 0.0311867 & 0.065 & 0.0988133 & 0.12 \\
$\mathrm{~F}$ & Resistor $[\Omega]$ & 0.40 & 0.997078 & 1.95 & 2.90292 & 3.50 \\
$\mathrm{G}$ & Inductor $[\mathrm{H}]$ & 0.001 & 0.020068 & 0.0505 & 0.080932 & 0.10 \\
\hline
\end{tabular}

allows us to analyse the system evolution without control system effects.

The model of the physic subsystem of power window is shown in Figure 4.

The model of power window system is characterized by independent and dependent parameters (Table 1). The independent parameters control the behaviour of the model. The change of independent parameters entails the variation of the lifting and/or lower law.

\section{Design of experiment within the power window system}

In order to build an empirical model for main responses, a sequential approach to experimentation, based on central composite design (CCD), was adopted aiming at characterizing and optimizing the system. A CCD is an experimental design which is often used in the response surface methodology (RSM) to build a second order (quadratic) model for the response variable. It contains an embedded factorial design with center points which are augmented with a group of 'star points' that allows an estimation of the curvature [16]. Within the set of independent parameters we take a subset as factors. We define just seven factors: A - window weight; B speed ratio; $\mathrm{C}$ - pulley radius; $\mathrm{D}$ - inertia; $\mathrm{E}$ - electromagnetic constant; $\mathrm{F}$ - resistor; $\mathrm{G}$ - inductor. As required by CCD, we determine factorial points $(+1$ and -1$)$, centre points $(0)$ and axial points $(+\alpha$ and $-\alpha)$. According to the characteristics of power window systems available on the market, we define the factor ranges in terms of alphas $(-\alpha,+\alpha)$. The values are shown in Table 2 . 
Design-Expert@ Software

Factor Coding: Actual

Stroke time (s)

- Design Points

.... $95 \% \mathrm{Cl}$ Bands

$\mathrm{X} 1=\mathrm{B}:$ Speed ratio

Actual Factors

A: Window weight $=4$

C: Pulley radius $=0.035$

$\mathrm{D}$ : Inertia (transmission) $=0.004$

$\mathrm{E}:$ K electromagnetic $=0.065$

$\mathrm{F}:$ Resistor $=1.95$

$\mathrm{G}$ : Inductor $=0.0505$

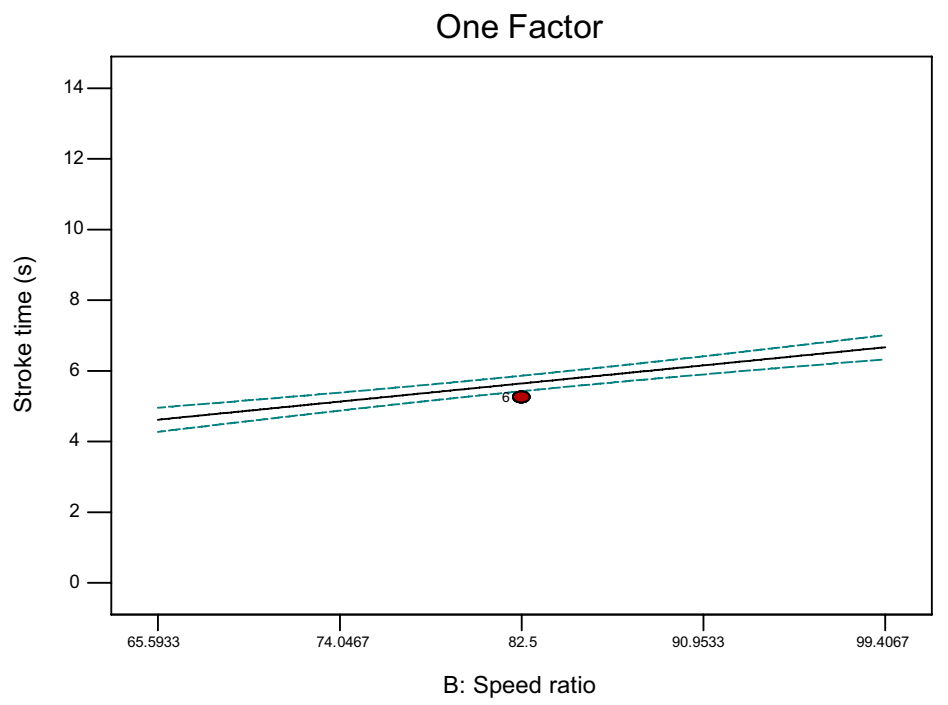

Design-Expert® Software

Factor Coding: Actua

Stroke time (s)

- Design points below predicted value

12.6

$\mathrm{X} 1=\mathrm{C}:$ Pulley radius

$\mathrm{X} 2=\mathrm{E}:$ K electromagnetic

Actual Factors

A: Window weight $=2$

B: Speed ratio $=82.5$

D: Inertia (transmission) $=0.004$

$\mathrm{F}:$ Resistor $=1.95$

$\mathrm{G}:$ Inductor $=0.0505$

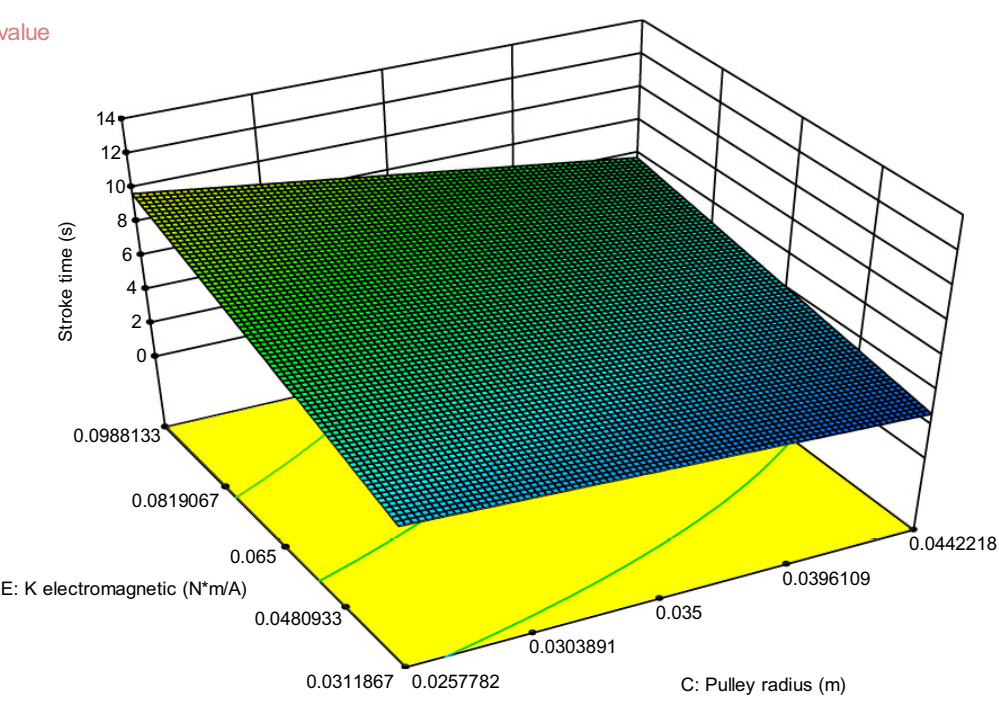

Fig. 5. Response surface of quadratic factor $C E$ when level for all factors is set to 0 .

The full factorial experiment is composed by 152 experimental runs. We carry out a fractional factorial experiment of 50 experimental runs, using 6 centre points and 44 not centre points.

In this paper, the stroke time of the window $[\mathrm{s}]$ is collected as main response (R1), being extremely significant as the user perceived only such response from the power window system. Other responses are necessary to perform the designing of power window system within the context of a specific car door.

\section{Results}

The results related to 50 experimental runs are briefly summed up in this section. In particular, the trend of stroke time is represented when the factors change.
ANOVA analysis for stroke time (R1) highlights that four factors are significant (linear factors are three; 2factor interaction is one): (1) $\mathrm{E}$ - electromagnetic constant; (2) $\mathrm{C}$ - pulley radius; (3) $\mathrm{B}$ - speed ratio; (4) $\mathrm{CE}$ pulley radius and electromagnetic constant interaction.

The model is significant and has a $R$-squared value of 0.9092 .

The final equation in terms of actual factors is:

$$
\begin{aligned}
R 1=-3.295+0.06 B & -10.085 C \\
& +137.959 E-2059.050 C E
\end{aligned}
$$

Equation (1) can be used to predict the physical system response according to a given set of values, in the defined range (just interpolation), for each factor (not necessarily a treatment combination). In particular, it presents an interaction between two significant factors $(C$ and $E)$. 
Table 3. Analysis of variance.

\begin{tabular}{cccccc}
\hline Source & Squares & df & Square & Value & Prob $>F$ \\
\hline Model & 288.66 & 35 & 8.25 & 29.10 & $<0.0001$ significant \\
$A$-Window weight & 0.54 & 1 & 0.54 & 1.91 & 0.1887 \\
$B$-Speed ratio & 24.96 & 1 & 24.96 & 88.06 & $<0.0001$ \\
$C$-Pulley radius & 45.50 & 1 & 45.50 & 160.57 & $<0.0001$ \\
$D$-Inertia (transmission) & $3.026 \mathrm{E}-003$ & 1 & $3.026 \mathrm{E}-003$ & 0.011 & 0.9192 \\
$E$ - Electromagnetic constant & 127.52 & 1 & 127.52 & 449.99 & $<0.0001$ \\
$F$-Resistor & 2.74 & 1 & 2.74 & 9.66 & 0.0077 \\
$G$-Inductor & 0.045 & 1 & 0.045 & 0.16 & 0.6957 \\
$A B$ & 0.20 & 1 & 0.20 & 0.70 & 0.4158 \\
$\ldots$ & $\ldots$ & $\ldots$ & $\ldots$ & $\ldots$ & $\ldots$ \\
$C D$ & $1.871 \mathrm{E}-003$ & 1 & $1.871 \mathrm{E}-003$ & $6.603 \mathrm{E}-003$ & 0.9364 \\
$C E$ & 8.91 & 1 & 8.91 & 31.43 & $<0.0001$ \\
$C F$ & $1.122 \mathrm{E}-003$ & 1 & $1.122 \mathrm{E}-003$ & $3.958 \mathrm{E}-003$ & 0.9507 \\
$\ldots$ & $\ldots$ & $\ldots$ & $\ldots$ & $\ldots$ & $\ldots$ \\
$G^{2}$ & 0.10 & 1 & 0.10 & 0.35 & 0.5619 \\
Residual & 3.97 & 14 & 0.28 & & \\
Lack of Fit & 3.97 & 9 & 0.44 & & \\
Pure Error & 0.000 & 5 & 0.000 & & \\
Cor Total & 292.63 & 49 & & & \\
\hline
\end{tabular}

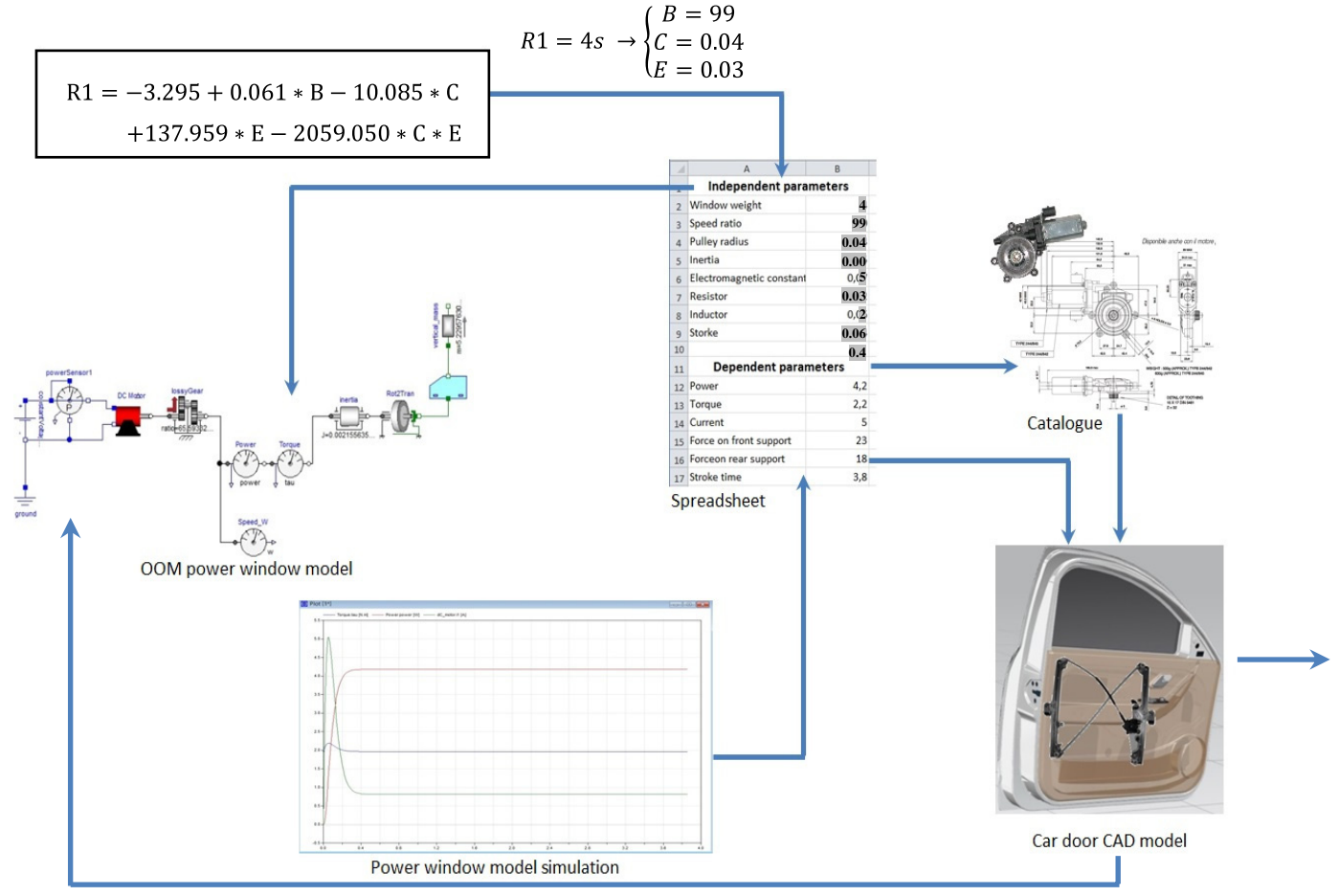

Fig. 6. Digital pattern data flow for the power window system.

\subsection{The digital pattern model of the power window system}

The release of geometrical and numerical models able to be easily adapted according to several sets of constraints i.e. the digital pattern model of the power window system is accomplished. Equation (1) is used to determine the factors that assure an expected response $R 1$. $B, C$ and $E$ control factors are used as independent parameters related to the first level while the other factors were placed at a secondary level. A car door 3D $\mathrm{CAD}$ model is linked to simulation data. A spread sheet acts as a buffer between OOM and CAD model of the car door. In such a way, Equation (1) is used to determine the obtainable configurations for a fixed stroke time. Then, the CAD model is used to verify different configurations, in integrated way, releasing the geometrically and physically acceptable configuration. Figure 6 depicts the data flow related to the digital pattern of power window system. 


\section{Conclusions and further works}

The paper deals with the designing of automotive power window by integrating OOM and DoE within a digital pattern approach. OOM and DoE in fact, are used as tools of a decision support system aimed to improve designers' tasks during the development process for power window systems. The Response Surface Method (RSM) with Central Composite Design (CCD) is used to identify the significant factors addressing the main system response, namely the stroke time. Then, the simulations of dependent parameters of the power window system, characterized by a double bowden sliding mechanism, are accomplished together with the data flow related to the product development loop. The equation that predicts the main response of the physical system, according to a given set of levels for each factor, expresses a quadratic dependency related to them. The OOM demonstrates the effective integration between different subsystems and related physics, solving a strategic challenge within the designing of mechatronic systems. Then, the parameters as well as the developed object models could be integrated in a graphical user interface to increase the accuracy of design activities and to reduce the development time to be spent for a new power window system.

Experimental tests performed on physical power window system are due to validate the mathematical models here accomplished.

Acknowledgements. The present work was developed with the economic support of MIUR (Italian Ministry of University and Research) performing the activities of the project PON01_01268 "Digital pattern product development: a pattern driven approach for industrial product design".

\section{References}

[1] W. Borutzky, Bond graph modelling and simulation of multidisciplinary systems - An introduction, Simul. Modell. Pract. Theory 17 (2009) 3-21

[2] M. Abramovici, F. Bellalouna, Service oriented architecture for the integration of domain-specific PLM systems within the mechatronic product development, in: Proceedings of the 7th International Symposium on Tools and Methods of Competitive Engineering (TMCE 2008), Izmir, Turkey, 2008, pp. 941-953
[3] C. Zheng, M. Bricogne, J. Le Duigou, B. Eynard, Survey on mechatronic engineering: a focus on design methods and product models, Adv. Eng. Info. 28 (2014) 241-257

[4] P. Hehenberger, Perspectives on hierarchical modeling in mechatronic design, Adv. Eng. Info. 28 (2014) 188-197

[5] C. Zheng, M. Bricogne, J. Le Duigou, B. Eynard, Mechatronic design process: a survey of product data model, procedia CIRP 21 (2014) $282-287$

[6] M. Tomizuka, Mechatronics: from the 20th to 21st century (plenary talk), Proc. of 1st IFAC Conference on Mechatronic Systems, 18-20 September, Darmstadt, Germany, 2000

[7] J. Lefévre, S. Charles, M. Bosch-Mauchand, B. Eynard, E. Padiolleau, Multidisciplinary modelling and simulation for mechatronic design, J. Design Res. 12 (2014) $127-144$

[8] J. Van Amerongen, P. Breedveld, Modelling of physical systems for the design and control of mechatronic systems, Ann. Rev. Control 27 (2013) 87-117

[9] B. Zupančič, A. Sodja, Advanced multi-domain modelling: advantages and dangers. UKSim 13th International Conference on Modelling and Simulation, 2011

[10] A. Lanzotti, S. Patalano, F. Vitolo, A Graph-based Software Tool for the CAD Modeling of Mechanical Assemblies, GRAPP 2013 IVAPP 2013, Proc. of 8th International Conference on Computer Graphics, Theory and Applications, Barcelona, Spain, 21th-24th February, SciTePress Digital Library, 2013, pp. 60-69

[11] S. Patalano, F. Vitolo, A. Lanzotti, A graph-based approach to CAD modeling: a digital pattern application to the sizing and modeling of manual transverse gearboxes. Proc. of International Conference on Graphic Engineering, Madrid, Spain, June 19th-21st, 2013

[12] S. Mustafiz, J. Joachim Denil, L. Lucio, H. Vangheluwe, The FTG+PM framework for multi-paradigm modelling: An automotive case study, in Proceedings of 6th International Workshop on Multi-Paradigm Modeling MPM'12, Innsbruck, Austria, 2012

[13] W.S. Ra, H.J. Lee, J.B. Park, Practical pinch detection algorithm for smart automotive power window control systems, IEEE Trans. Ind. Electron. 55 (2008) 1376-1384

[14] R. Chhabra, M. Reza Emami, Holistic system modeling in mechatronics, Mechatronics 21 (2001) 166-175

[15] S.M. Prabhu, P.J. Mosterman, Modelling, Simulating, and Validating a Power Window System Using a Model-Based Design Approach. Automotive Digest, The MathWorks, 11/2003

[16] Box Hunter: Statistics for Experimenters: Design, Innovation, and Discovery, 2nd Edition 